\title{
A durable design
}

\section{Vaccines on the market aren't practical for the developing world - where cervical cancer hits hardest - but researchers are trying to make ones that are.}

\section{BY KATHARINE SANDERSON}

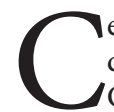

ervical cancer is most deadly in the developing world. According to the GAVI Alliance, a group of leading organizations working to improve access to immunization, $88 \%$ of the 275,008 women who died in 2008 from cervical cancer lived in developing countries.

At least 15 types of human papillomavirus (HPV) are implicated in cervical cancer. The two vaccines on the market, Merck's Gardasil and GlaxoSmithKline's Cervarix, offer protection against the most prevalent types, HPV 16 and HPV 18, which between them cause $70 \%$ of cervical cancer cases (see 'The global burden', page S2). But, for several reasons, these vaccines are not ideal.

Cost is an impediment. Susan Wang, a medical officer in the Expanded Programme for Immunization (EPI) at the World Health Organization (WHO) in Geneva, estimates that it costs between US $\$ 4$ and $\$ 6$ to vaccinate each girl, money that many developing countries can ill afford.

$\omega_{\Sigma}^{\infty}$ Developing countries also face huge logistical hurdles. Vaccines need cold storage to be viable, but this is difficult in regions with hot climates and unreliable electricity. Then there's the problem of ensuring the right people receive the right doses. Both vaccines require 3 injected doses over 6 months; in remote areas and in cultures where the need for vaccinations are poorly understood, this schedule is difficult to ensure.

The current vaccines that target HPV 16/18 do not protect against $30 \%$ of cervical cancer cases. What's more, if HPV 16/18 are suppressed, there is a risk that other HPV types will proliferate in their place (see 'Trials of an anticancer jab', page S4). A vaccine offering broader protection against all cancer-causing strains is the objective.

What's more, prophylactic vaccines are useless for someone who is already infected. A therapeutic jab that could eliminate all HPV infection plus any cancerous cells would enable a far more effective vaccination programme in combating cervical cancer.

Next-generation HPV vaccines are in development. Scientists are targeting different proteins with the ultimate goal of developing a single-dose vaccine effective against more types of the virus, which is more durable and cheaper to make.
One strategy is to tweak the current generation of vaccines. Gardasil and Cervarix both use the L1 proteins that coat specific HPV types to make virus-like particles (VLPs). It is VLPs that trick the immune system into reacting to a viral intruder, triggering the production of antibodies that subsequently protect against HPV infection.

Gardasil includes four L1 proteins, Cervarix two. To broaden coverage, Merck is developing a vaccine that includes nine viral proteins. But this



Darin Wick (foreground) and John Webb hold some HPV-infected tumour cells up to the light.

vaccine is likely to be expensive because it relies on existing, costly manufacturing methods. Virologist Lutz Gissmann from the German Cancer Research Centre in Heidelberg says that bombarding the body with so many antigen -provoking proteins can tire the immune system: there are instances (not in HPV) where adding an extra antigen to a vaccine lowers the immunogenicity of the others, he adds.

\section{LOCAL PRODUCE}

As an alternative approach, pathologist Richard Roden at Johns Hopkins University in Baltimore, Maryland, exploits another viral protein, L2. These L2 proteins stimulate a weaker immune response than L1 proteins, but one that is active against more types of HPV. Roden's group fuses together units of L2 from various HPV types to make a single polypeptide chain that can be expressed by Escherichia coli (not possible with L1 proteins). Roden contends that using bacteria to produce single polypeptides would simplify manufacturing to such an extent that the vaccine could be produced in local laboratories, bringing costs down significantly. The L2 vaccine is due to enter phase I clinical trials in early 2013. "This is fantastic," comments Gissmann, who is not involved with the work. "It's a completely different principle and I think it's doable."

Other researchers are pursuing therapeutic angles. Immunologist John Webb at the $\mathrm{BC}$ Cancer Agency in Victoria, Canada, is developing a vaccine that targets $\mathrm{E} 7$ proteins. E proteins activate once HPV has infected a cell. They promote the growth of cancer cells by inactivating tumour suppressing proteins.

Antibodies are useless against E7 because, by this stage, the virus is inside the cell. Instead, an immune cell, $\mathrm{CD}^{+}$cells, that kills virusinfected cells is the best form of defence. Past attempts to trigger CD8 responses have targeted particular disease-causing protein subunits. According to Webb, these techniques "work - but very inefficiently" because the subunits are so specific that the virus can easily evade the immune response. Webb's vaccine instead contains whole E7 proteins from the five most common cancer-causing HPV types, as well as poly(I:C) - a double-stranded RNA molecule that signals the presence of a virus. Together these molecules stimulate a swift build-up of CD8. In mice, administering the vaccine once a day for 4 days caused tumours to shrink often disappearing — within 3 weeks (Wick, D. A. \& Webb, J. R. Vaccine 29, 7857-7866, 2011). Webb speculates that this concentrated exposure to two simultaneous agents helps the vaccine "emulate the effect of a true viral infection" and hence strengthens the result.

What's more, the whole-protein formulation of this vaccine, called Pentarix, makes it more stable than the minimal peptide approaches, and without the need for refrigeration, says Webb. "It may be better suited to developingworld settings than prophylactic vaccines."

Gissmann is optimistic that new vaccines will soon start to show success in clinical trials, solving some of the problems that have beset HPV vaccines on the market. So the next generation of HPV vaccines might finally be able to reach the people who need them most.

Katharine Sanderson is a freelance journalist based in Toulouse, France. 